\title{
Die Zwangserkrankung im Kontext psychiatrischer Versorgung
}

\author{
Obsessive Compulsive Disorder in Psychiatric Care
}

Autor

Institut
Katarina Stengler

Leiterin Allgemeinambulanz und Ambulanz für Zwangserkrankungen, Klinik und Poliklinik für Psychiatrie und Psychotherapie, Universitätsklinikum Leipzig

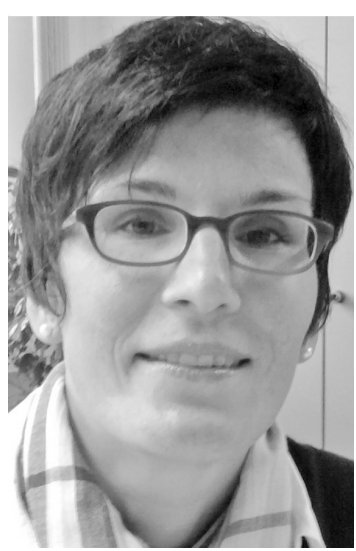

Priv.-Doz. Dr. med. Katarina Stengler

Bibliografie

DOI http://dx.doi.org/

10.1055/s-0030-1248626

Psychiat Prax 2010; 37 :

363-365

(c) Georg Thieme Verlag KG Stuttgart · New York .

ISSN 0303-4259

Korrespondenzadresse

Priv.-Doz. Dr. med.

Katarina Stengler

Leiterin Allgemeinambulanz und Ambulanz für Zwangserkrankungen, Klinik und Poliklinik für Psychiatrie,

Universitätsklinikum Leipzig

Semmelweisstraße 10

04103 Leipzig

Katarina.Stengler@medizin.unileipzig.de

\section{Zwangserkrankungen sind folgenschwer} $\nabla$

Zwangserkrankungen gehören mit einer Lebenszeitprävalenz von ca. 2-2,5\% zu den häufigsten psychischen Störungen und zählen nach einer von der WHO und Weltbank in Auftrag gegebenen Studie zu den 10 Erkrankungen, die mit den größten psychosozialen Behinderungen einhergehen [1].

Die aktuelle mit dem ICD-10 oder DSM-IV verbundene operationalisierte Diagnostik und deskriptive, den psychopathologischen Symptomkonstellationen folgende Klassifikation verzichtet bekanntermaßen auf ätiopathogenetische $\mathrm{Zu}$ schreibungen. Mit zunehmender Kenntnis der phänomenologischen Heterogenität der Zwangserkrankung [2] wurde in den letzten Jahren die klassische Definition der reinen Zwangsstörung konzeptuell um Forschungsansätze zur Beschreibung von Subtypen erweitert. Hierbei werden neben phänomenologischen Aspekten (relativ konsistent beschriebene Symptomcluster) weitere klinische und andere Parameter, wie etwa Geschlecht, Alter bei Krankheitsausbruch und Komorbidität berücksichtigt [3]. Stärker konzentriert sich gegenwärtig dabei die Ursachenforschung auf neurobiologische Aspekte mit Fokus auf familiäre und damit genetisch bedingte Häufungsmuster bei bestimmten Untergruppen der Zwangserkrankung [4].

\section{Frühverlauf und Unterversorgung $\nabla$}

Unbehandelte psychische Erkrankungen verlaufen häufig chronisch und können zunehmend mit psychosozialen Behinderungen und somatischen Komplikationen einher gehen [5]. Es verwundert deshalb nicht, wenn in den letzten Jahren die Forschung zur Früherkennung psychischer Erkrankungen mit dem Fokus auf mög- lichst frühzeitige Diagnostik und Therapie, intensiviert wurde. Bisherige Untersuchungen konzentrierten sich dabei insbesondere auf die Schizophrenie [6,7], auf Depressionen [8] und auf Angsterkrankungen [9].

Nur wenig ist dagegen bekannt über den Frühverlauf von Zwangserkrankungen. Untersuchungen zur Heterogenität der Zwangserkrankung [10] und Verlaufsstudien mit zwangserkrankten Kindern und Jugendlichen haben jedoch gezeigt, dass ein früher Erkrankungsbeginn mit schlechteren Therapieresponseraten und einer ungünstigen Langzeitprognose einhergehen kann [3].

Unspezifische prodromale Auffälligkeiten die, wie im Falle der Schizophrenie viele Jahre vor manifestem Krankheitsausbruch bereits zu Einschränkungen führen können, wurden in bisherigen Studien bei Zwangserkrankungen kaum erfasst. Stengler-Wenzke u. Angermeyer [11] konnten in einer kleineren qualitativen Untersuchung zeigen, dass erste zwangsähnliche Auffälligkeiten von den Patienten zwar frühzeitig wahrgenommen, dann aber banalisiert bzw. einer im elterlichen Erziehungsstil antizipierten Norm zugeordnet und nur in den wenigsten Fällen als „krank“ eingeschätzt wurden. Nach Angaben der Betroffenen verfahren Angehörige in der Regel ebenso: die ihnen anvertrauten oder von ihnen bemerkten „Auffälligkeiten“ werden bagatellisiert oder familiären Verhaltensmustern zugeschrieben. Das Aufsuchen professioneller Hilfe als Ausdruck einer bereits so definierten „Krankheit“ war die Ausnahme, verzögerte Inanspruchnahme therapeutischer Hilfe aber eher die Regel.

Die Tatsache, dass Patienten mit Zwangserkrankungen im Mittel 7-10 Jahre nach dem Auftreten von Zwangssymptomen erstmals therapeutische Hilfe in Anspruch nehmen [12] und zu diesem Zeitpunkt oft schon ein hoher Grad an Chronifizierung $[13,14]$ mit zumeist somatischen und sozialmedizinisch relevanten Folgeschäden einge- 
treten ist, unterstreicht die Notwendigkeit der Früherkennung und -behandlung von Zwangserkrankungen.

Die Bedingungen und Ursachen, die zu einer späten Inanspruchnahme von professioneller Hilfe bei Zwangserkrankungen führen, wurden bislang wenig untersucht. In der oben erwähnten qualitativen Studie [11] kritisierten die Befragten mangelndes Wissen der Professionellen. Defizite in der psychiatrisch-professionellen Versorgung thematisierten sie insbesondere dort, wo häufige Fehldiagnosen gestellt oder die von ihnen vorgetragenen Beschwerden banalisiert wurden. Sie sahen sich zudem nicht ausreichend über spezifische Unterstützungsangebote informiert.

Mangelndes Wissen um adäquate professionelle Hilfe erfassten Goodwin et al. [15] in einer Stichtagserhebung als wesentlichen Prädiktor für nicht in Anspruch genommene Behandlung bei Patienten mit Zwangserkrankungen. Besiroglu et al. [16] wiesen ebenfalls auf die Bedeutung des vorbestehenden Wissens im Zusammenhang mit Inanspruchnahme von professioneller Hilfe hin, als sie Patienten mit Zwangserkrankungen untersuchten, die keine Therapie und solche, die eine in Anspruch genommen hatten.

Von Wahl et al. [17] wurde die Bedeutung der frühzeitigen Diagnosestellung für die Inanspruchnahme therapeutischer Angebote jüngst für Deutschland unterstrichen: über 70\% der befragten Zwangserkrankten waren in der ambulanten Versorgung als solche nicht erkannt worden und blieben folglich ohne diagnosespezifische Behandlung. Ebenso katastrophal sieht die Versorgungssituation von Zwangserkrankten aus, wenn man die professionelle Seite befragt. In einer auch kürzlich publizierten Studie von Külz u. Mitarb. [18] wurden psychologische und ärztliche Psychotherapeuten in Süddeutschland mithilfe eines Fragebogens zu Art, Häufigkeit und konkreter Durchführung der (psychotherapeutischen) Behandlung von Zwangserkrankten befragt. Bei über $80 \%$ der Befragten spielte die Behandlung von Zwangserkrankten keine oder eine sehr geringe Rolle.

Scham, Peinlichkeit und daraus resultierende Geheimhaltung der Symptomatik scheinen ebenso zu einer späten Inanspruchnahme von professioneller Hilfe bei Zwangserkrankten beizutragen. Verschweigen der Auffälligkeiten wurde von Zwangserkrankten (und auch deren Angehörigen) in einer eigenen Untersuchung [19] als wichtigste Bewältigungsstrategie für Stigmatisierung angegeben. Hier war insbesondere die antizipierte Stigmatisierung bedeutsam: je mehr die Zwangserkrankten mit Ablehnung im privaten und öffentlichen Umfeld rechneten, desto stärker tendierten sie zur Geheimhaltung. Dies führt wohl kurzfristig zur Entlastung der Betroffenen, langfristig trägt es unweigerlich zur Aufrechterhaltung des dysfunktionalen Systems und insbesondere zur Verhinderung frühzeitiger Diagnostik und Behandlung der Zwangsstörung bei.

\section{Desiderate für Versorgung und Forschung \\ $\nabla$}

Der Mangel an Informationen und an Wissen über die Erkrankung ist ein wesentliches Hindernis zur Inanspruchnahme professioneller Hilfe bei Zwangserkrankungen [20,21]. Dem gilt es durch umfangreiche psychoedukative Maßnahmen konsequent zu begegnen. Die Furcht vor Stigma und deren Bewältigung durch Geheimhaltung sind für Zwangserkrankte weitere Barrieren, Behandlung aufzunehmen. Dabei scheint die Angst vor Eti- kettierung, vor „Sichtbar-machen des Makels“ als stärkster Auslöser öffentlicher Stigmatisierung [22] bestimmend zu sein, denn Aufnahme von psychiatrischer Behandlung hieße, sich als Zwangserkrankter etikettieren zu lassen. Es gilt also, erfolgreiche Stigmabewältigung als unterstützende Maßnahme zur (frühzeitigen) Inanspruchnahme von professioneller Hilfe zu verstehen. Dem sollte auf struktureller Ebene genauso Rechnung getragen werden, wie auf individuell-psychologischer. Wünschenswert ist demnach, jenseits der diagnosespezifischen Spezialsprechstundenangebote, im medizinischen Versorgungsalltag, ob beim Fach- oder Hausarzt, entsprechend niedrigschwellige Behandlungsalternativen zu schaffen.

Die von Thornicroft u. Tansella [23] beschriebenen „components of a modern mental health service“ favorisieren ein stufenweises Vorgehen innerhalb des psychiatrischen Versorgungssystems in Abhängigkeit von den jeweils verfügbaren Ressourcen. Damit wird nicht nur die Hoffnung auf eine ökonomischere Vorgehensweise verbunden, sondern auch ein für die jeweilige Versorgungsstufe inhaltlich adäquates Behandlungsangebot versprochen. Die aktuell in Entwicklung befindliche S3-Leitlinie für Zwangserkrankungen könnte hier, ähnlich wie die NICE-Guidelines, mit der Formulierung eines stepped care models ein wichtiger Wegweiser sein. In der psychiatrischen Versorgung von Zwangserkrankten sollte zukünftig stärker die Rolle der Angehörigen, insbesondere in der psychoedukativen Arbeit berücksichtigt werden. Angehörige brauchen Informationen, wenn sie mit ersten Auffälligkeiten im Frühverlauf der Zwangserkrankung der nicht selten im Kindesalter liegen kann - konfrontiert werden. Wissen über veränderte Verhaltensweisen kann Verständnis wecken und eine frühe Behandlungsaufnahme unterstützen. Der Forschungsbedarf an Studien zur frühen Identifikation von Zwangserkrankungen ist aufgrund mangelnder „Objektivierbarkeit" des tatsächlichen Krankheitsausbruches nicht gedeckt. Die Entwicklung eines Symptominventars, das neben diesen retrospektiv erfassten frühen Auffälligkeiten, ergänzende Informationen der Angehörigen und „objektive“ Kriterien der Professionellen enthält, ist notwendig. Durch eine frühzeitige Diagnosenstellung kann somit eine zeitnahe Indikationsprüfung zur gezielten Behandlungsaufnahme erreicht werden.

\section{Literatur}

1 Lopez AD, Murray CC. The global burden of disease, 1990-2020. Nat Med 1998; 4: 1241-1243

2 Fontenelle LF, Hasler G. The analytical epidemiology of obsessive-compulsive disorder: risk factors and correlates. Prog Neuropsychopharmacol Biol Psychiatry 2008; 32: 1-15

3 Fontenelle LF, Mendlowicz MV, Marques C et al. Early- and late-onset obsessive-compulsive disorder in adult patients: an exploratory clinical and therapeutic study. J Psychiatr Res 2003; 37: 127-133

4 Dold $M$, Aigner $M$. Neuroimaging the various symptom dimensions of obsessive-compulsive disorder. Neuropsychiatr 2009; 23: 193-205

5 Häfner $H$. Prevention and early intervention in schizophrenia: facts and visions. Seishin Shinkeigaku Zasshi 2002; 104: 1033-1054

6 Häfner H. Das Rätsel Schizophrenie. Eine Krankheit wird entschlüsselt. München: C. H. Beck; 2000: 396-408

7 McGorry PD, Yung AR. Early intervention in psychosis: an overdue reform. Aust N Z J Psychiatry 2003; 37: 393-398

8 Lewinsohn PM, Pettit JW, Joiner Jr TE et al. The symptomatic expression of major depressive disorder in adolescents and young adults. J Abnorm Psychol 2003; 112: 244-252

9 Wittchen $\mathrm{HU}$, Fehm L. Epidemiology and natural course of social fears and social phobia. Acta Psychiatr Scand 2003; 417: 4-18

10 Lochner C, Stein DJ. Heterogeneity of obsessive-compulsive disorder: a literature review. Harv Rev Psychiatry 2003; 11: 113-132 
11 Stengler-Wenzke K, Angermeyer MC. Employment of professional help by patients with obsessive-compulsive disorders. Psychiat Prax 2005; 32: 195-201

12 Grabe HJ, Meyer C, Hapke U et al. Prevalence, quality of life and psychosocial function in obsessive-compulsive disorder and subclinical obsessive-compulsive disorder in northern Germany. Eur Arch Psychiatry Clin Neurosci 2000; 250: 262-268

13 Skoog G, Skoog I. A 40-year follow-up of patients with obsessive-compulsive disorder. Arch Gen Psychiatry 1999; 56: 121-127

14 Angst J, Gamma A, Endrass J et al. Obsessive-compulsive severity spectrum in the community: prevalence, comorbidity, and course. Eur Arch Psychiatry Clin Neurosci 2004; 254: 156-164

15 Goodwin R, Koenen KC, Hellman F et al. Helpseeking and access to mental health treatment for obsessive-compulsive disorder. Acta Psychiatr Scand 2002; 106: 143-149

16 Besiroglu L, CIlli AS, Askin R. The predictors of health care seeking behavior in obsessive-compulsive disorder. Compr Psychiatry 2004; 45: 99-108

17 Wahl K, Kordon A, Kuelz KA et al. Obsessive-Compulsive Disorder (OCD) is still an unrecognised disorder: A study on the recognition of OCD in psychiatric outpatients. Eur Psychiatry 2010 e-first: doi 10.1016/j. eurpsy.2009.12.003

18 Külz AK, Hassenpflug K, Riemann D et al. Psychotherapeutic care in OCD outpatients - results from an anonymous therapist survey. Psychother Psych Med 2010; 60: 194-201

19 Stengler-Wenzke K, Beck M, Holzinger A et al. Stigma experiences of patients with obsessive compulsive disorders. Fortschr Neurol Psychiatr 2004; 72: 7-13

20 Riedel-Heller SG. Research in social psychiatry - addressing future challenges of health- and social systems. Neuropsychiatr 2009; 23: 249252

21 Hinterhuber H, Meise $U$. No psychiatry without social psychiatry. Neuropsychiatr 2008; 22: 148-152

22 Link BG, Cullen FT, Frank J et al. The social rejection of former mental patients: understanding why labels matter. Am J Soc 1987; 92: 14611500

23 Thornicroft G, Tansella $M$. Components of a modern mental health service: a pragmatic balance of community and hospital care: overview of systematic evidence. Br J Psychiatry 2004; 185: 283-290 